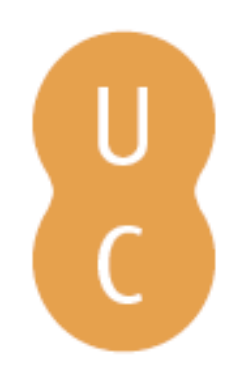

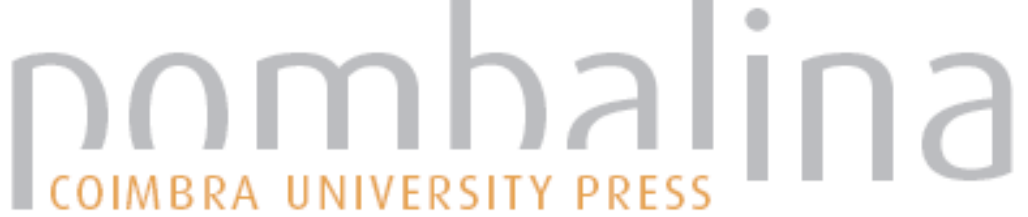

\section{Determinação do índice de qualidade das águas do rio São Paulo, Candeias, Bahia,} Brasil

\author{
Autor(es): \\ Ramos Junior, A. \\ B. S.; Cruz, M. J. M.; Santos, R. A.; Gonçalves, M. V. \\ P.
}

Publicado por: Imprensa da Universidade de Coimbra

URL persistente:

URI:http://hdl.handle.net/10316.2/31442

DOI:

DOl:http://dx.doi.org/10.14195/978-989-26-0531-9_17

Accessed : $\quad$ 26-Apr-2023 14:06:57

A navegação consulta e descarregamento dos títulos inseridos nas Bibliotecas Digitais UC Digitalis, UC Pombalina e UC Impactum, pressupõem a aceitação plena e sem reservas dos Termos e Condições de Uso destas Bibliotecas Digitais, disponíveis em https://digitalis.uc.pt/pt-pt/termos.

Conforme exposto nos referidos Termos e Condições de Uso, o descarregamento de títulos de acesso restrito requer uma licença válida de autorização devendo o utilizador aceder ao(s) documento(s) a partir de um endereço de IP da instituição detentora da supramencionada licença.

Ao utilizador é apenas permitido o descarregamento para uso pessoal, pelo que o emprego do(s) título(s) descarregado(s) para outro fim, designadamente comercial, carece de autorização do respetivo autor ou editor da obra.

Na medida em que todas as obras da UC Digitalis se encontram protegidas pelo Código do Direito de Autor e Direitos Conexos e demais legislação aplicável, toda a cópia, parcial ou total, deste documento, nos casos em que é legalmente admitida, deverá conter ou fazer-se acompanhar por este aviso.

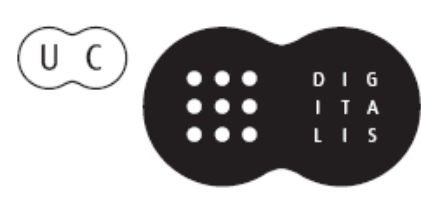





\title{
DETERMINAÇÁO DO ÍNDICE DE QUALIDADE DAS ÁGUAS DO RIO SÁO PAULO, CANDEIAS, BAHIA, BRASIL
}

\author{
DETERMINATION OF WATER QUALITY INDEX OF SAO PAULO \\ RIVER, CANDEIAS, BAHIA, BRAZIL
}

A. B. S. Ramos Junior ${ }^{1}$, M. J. M. Cruz², R. A. Santos ${ }^{1} \&$ M. V. P. Gonçalves ${ }^{1}$

\begin{abstract}
Resumo - Os impactos ambientais gerados por efluentes nos cursos d'agua têm crescido de maneira alarmante, especialmente em grandes centros urbanos, isso em função da deficiência da rede de esgoto sanitário e baixa conscientizaçáo da população em relaçáo à conservação dos corpos hídricos. A necessidade de um maior conhecimento e controle da variabilidade temporal e espacial desse tipo de impacto levou ao desenvolvimento de índices de qualidade das águas (IQA) que é calculado pelo produtório ponderado das qualidades de água correspondentes às variáveis que integram o índice e reflete a interferência de substâncias orgânicas, nutrientes, sólidos e microbiológicos na qualidade das águas para consumo humano. Visto que a região do rio São Paulo-BA é vulnerável às contaminaçôes domésticas e industriais, o presente trabalho objetiva determinar o Índice de Qualidade das Águas, estabelecendo a variabilidade espacial da qualidade, a fim de possibilitar uma melhor integração e interpretação dos dados. A coleta foi realizada em um período caracterizado por chuvas esparsas em dez pontos, na superfície e ao longo do Rio Sáo Paulo onde foram determinados parâmetros físicos, químicos e biológicos. Os valores de IQA determinados nos dez pontos amostrados revelam que as águas do Rio São Paulo estáo classificadas de regular a boa e o IQA foi satisfatório para uma avaliação hidrogeoquímica do rio São Paulo, porém expressa de uma forma simplificada uma condição momentânea. Entretanto, O IQA pode ser utilizado para implementação de políticas de gerenciamentos dos Recursos Hídricos se for estabelecido um sistema de monitoramento.
\end{abstract}

Palavras-chave - Índice de Qualidade de Água, Rio São Paulo, Hidrogeoquímica.

${ }^{1}$ Programa de Pós-Graduação em Geologia, Universidade Federal da Bahia (UFBA), Salvador, Bahia, Brasil: bomfilhojr@yahoo.com.br, rodrigo.santos@ufba.br,zoovitor81@yahoo.com.br

${ }^{2}$ Dep. de Geoquímica, Instituto de Geociências da Universidade Federal da Bahia (UFBA), Salvador, Bahia, Brasil: jeronimo@ufba.br 
Abstract - The environmental impacts caused by effluents in rivers have increased alarmingly, especially in large urban centers, that the disability of the sewage network, and low public awareness regarding the conservation of water bodies. The need for greater knowledge and control of temporal and spatial variability of this type of impact, led to the development of water quality index (IQA) that is calculated by weighted multiplicand of the water qualities corresponding to the variables that comprise the index and reflects the interference from organic substances, nutrients, solids and microbiological quality of drinking water. Since the region of São Paulo river, Bahia is vulnerable to domestic and industrial contamination, this study aims to determine the water quality index, establishing the spatial variability of quality in order to enable better integration and interpretation of data. The data were collected in a period characterized by frequent showers, in ten points on the surface and along the São Paulo river, where certain physical, chemical and biological parameters were determined. The IQA values determined in ten sample locations shows that the waters of the São Paulo river are classified from regular to good, beeing the IQA suitable for an hydrogeochemical evaluation, but expressed in a simplified form a momentary condition. However, the AQI can be used for implementation of policies for managing water resources if a monitoring system is established.

Keywords - Water quality index (IQA), River São Paulo, Hydrogeochemistry.

\section{1 - Introdução}

As atividades antrópicas lançam diversos poluentes industriais na região, dos quais podemos destacar a presença de metais pesados, elementos de alta densidade com potenciais riscos ao meio ambiente e muitos deles venenosos a espécie humana (PEKEI et al., 2004).

Os impactos ambientais gerados por efluentes nos cursos d'agua têm crescido de maneira alarmante, especialmente em grandes centros urbanos, isso em função da deficiência da rede de esgoto sanitário e baixa conscientização da população em relação à conservação dos corpos hídricos (CETESB, 2003).

Os efeitos combinados da urbanização e das demais atividades antropogênicas associadas ao rápido crescimento populacional das últimas décadas são facilmente visualizados nos ecossistemas (THORNE \& WILLIAMS 1997; POMPEU et al., 2005). Muitos rios, lagos e reservatórios têm sido prejudicados como consequência do aumento de atividades humanas. Sendo assim, o planejamento e gestão dos recursos hídricos dependem de informaçóes confiáveis, tanto no que diz respeito à demanda como à oferta de água (BRAGA \& TUCCI,1999).

A necessidade de um maior conhecimento e controle da variabilidade temporal e espacial desse tipo de impacto levou ao desenvolvimento de índices de qualidade das águas (IQA). A idéia básica dos índices de qualidade é agrupar uma série de variáveis numa escala comum, combinando-as em um único número (ALMEIDA \& SCHWARZBOLD, 2003) que possa ser empregado pelos órgãos governamentais competentes para gerenciar a qualidade das águas de uma bacia hidrográfica (SIMÓES et al., 2007).

A elaboração de um IQA deve considerar apenas variáveis ambientais críticas que afetam determinado recurso hídrico, em função do tipo de uso e ocupação do solo. O primeiro IQA utilizado foi desenvolvido pela "National Foundation Sanitation (NFS)" 
dos Estados Unidos e baseia-se na análise de nove parâmetros: coliformes fecais, $\mathrm{pH}$, demanda bioquímica de oxigênio, nitrogênio total, fósforo total, temperatura, turbidez, resíduo total e oxigênio dissolvido, os quais são indicadores de poluição por efluentes domésticos (CETESB, 2004).

O IQA é calculado pelo produtório ponderado das qualidades de água correspondentes às variáveis que integram o índice e reflete a interferência de substâncias orgânicas, nutrientes, sólidos e microbiológicos na qualidade das águas para consumo humano. Vem sendo aplicado em uma escala crescente como ferramenta de avaliação da qualidade das águas (COMISTESINOS, 1990; ALMEIDA \& SCHWARZBOLD, 2003; MELO JÚNIOR et al., 2003; ANDRADE et al., 2005).

A região do rio São Paulo é vulnerável às contaminaçôes domésticas e industriais, e diante disso o presente trabalho teve por objetivo determinar o Índice de Qualidade das Águas, estabelecendo a variabilidade espacial da qualidade, a fim de possibilitar uma melhor integração e interpretação dos dados.

\section{2 - Área de estudo}

A Bahia, o maior estado da regiāo nordeste, abrange uma área de $561.026 \mathrm{~km}^{2}$, e possui a maior extensão costeira do Brasil, com cerca de $1.200 \mathrm{~km}$ de costa atlântica. Nesse estado se encontra o estuário do rio São Paulo, localizado no município de Candeias, que integra a região metropolitana de Salvador, no recôncavo baiano, porção interna superior da Baía de Todos os Santos.

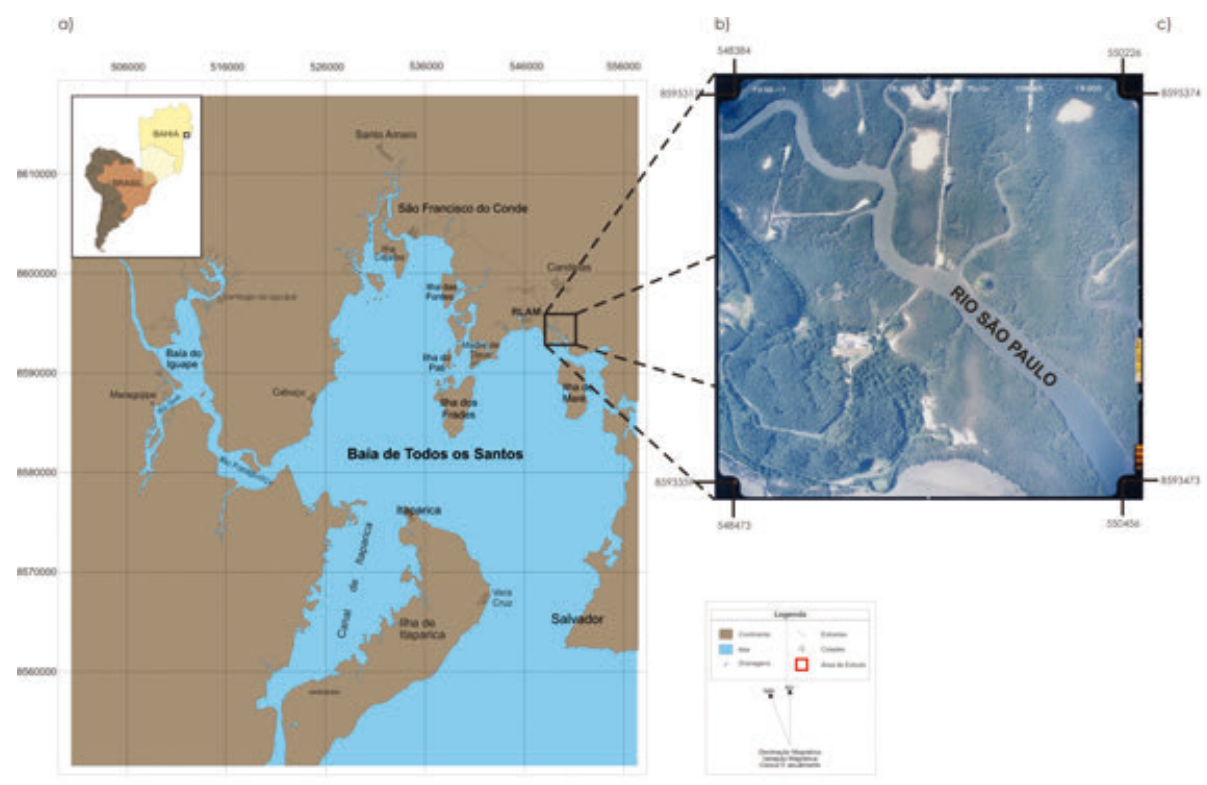

Fig. 1- Mapa de situação e localização do rio São Paulo, município de Candeias, Bahia, com imagem de satélite. 


\section{3 - Material e métodos}

Inicialmente foi feito um levantamento utilizando-se imagens de satélites e mapas topográficos.

A coleta foi realizada em um período caracterizado por chuvas esparsas (outubro/2011) em dez pontos, na superfície e ao longo do Rio São Paulo. Para tal coleta utilizou-se garrafas específicas para cada parâmetro a ser determinado, seguindo o APHA (2005).

Baseado nas características hidrográficas e hidrológicas (padrôes de drenagem, vazão, fluxo, presença de afluente, etc...) estabeleceu-se os dez pontos de amostragens, sendo o ponto 01 localizado a montante, até onde foi possível navegar, e o ponto 10 localizado já na desembocadura do Rio São Paulo com a Baía do Todos os Santos, totalizando uma extensão de $6 \mathrm{~km}$, e mantendo um intervalo de $600 \mathrm{~m}$ entre os pontos amostrais.

Foram determinados os seguintes parâmetros: Temperatura, Oxigênio Dissolvido (OD), Coliformes Termotolerantes, Demanda Bioquímica de Oxigênio (DBO), Fósforo (P), Nitrato $\left(\mathrm{NO}_{3}\right)$, pH, Sólidos Totais Dissolvido (STD) e Turbidez, alguns mensurados in situ com o auxílio de uma sonda multiparâmetro (Horiba) e outros determinados no laboratório, seguindo o APHA (2005).

Os nove parâmetros obtidos foram necessários para realização dos cálculos de IQA, através da fórmula;

$$
I Q A=\prod_{i=0}^{9} q_{i}^{w_{i}}
$$

onde: IQA: Índice de Qualidade das Águas, um número entre 0 e 100; qi: qualidade do i-ésimo parâmetro, um número entre 0 e 100, obtido da respectiva "curva média de variação de qualidade", em função de sua concentração ou medida e, wi: peso correspondente ao i-ésimo parâmetro, um número entre 0 e 1 , atribuído em função da sua importância para a conformação global de qualidade, sendo que:

$$
\sum_{i=1}^{n} w_{i}=1
$$

em que:

n: número de variáveis que entram no cálculo do IQA. Porém, todos os cálculos foram desenvolvidos através do software IQAIDATA, versão 2010 (UNISC), levando em consideração os pesos e a classificação proposta pela CETESB.

As análises estatísticas foram realizadas através do softwar Multi-Variate Statistical PacKage, version 3.2, já as análises geoestatísticas foram desenvolvidas com a utilização do programa computacional ArcGIS 9.3.

\section{4 - Resultados e discussão}

Os valores de IQA determinados nos dez pontos amostrados revelam que as águas do Rio São Paulo estão classificadas de regular a boas, seguindo o quadro de classificação proposto pela CETESB. 
Quadro 1 - Classificação do IQA.

\begin{tabular}{|c|c|}
\hline Categoria & Ponderaçäo \\
\hline ÓTIMA & $79<I Q A \leq 100$ \\
\hline BOA & $51<I Q A \leq 79$ \\
\hline REGULAR & $36<I Q A \leq 51$ \\
\hline RUIM & $19<I Q A \leq 36$ \\
\hline PÉSSIMA & $I Q A \leq 19$ \\
\hline
\end{tabular}

Os valores de IQA determinados foram caracterizados pela integração dos nove parâmetros, sendo alguns mais representativos que outros e se assemelham com valores encontrados em outros trabalhos ao estudarem áreas similares (ANDRADE et al., 2005; KONIG, 2008; STOLFI \& FIGUEIREDO, 2008; LOPES et al., 2008; PIASENTIN et al., 2009).

Foi possível verificar em cada ponto a representatividade dos parâmetros, sendo facilmente notável a influência desses parâmetros na determinação do Índice de Qualidade de Água. No qual podemos destacar os seguintes parâmetros como os mais representativos: Coliformes Termotolerantes, Turbidez e Oxigênio Dissolvido Saturado.

A análise de Cluster realizada pelo método de Ward classifica os parâmetros e os pontos de acordo com características peculiares entre si. Podendo desta forma, verificar se os parâmetros destacados como mais representativos, são os que realmente influenciam na determinação do IQA e distingui os diversos pontos por grupos.

O dendograma dos parâmetros pelo método Ward (Fig. 2) descreve três grupos diferentes: $\mathrm{O}$ grupo 1 caracterizado por inferior é representado pelos Coliformes Termotolerantes (COL), parâmetro considerado o mais representativo, agindo como principal agente na classificação do IQA, sendo possível notar que os pontos que possuem o maior número de COL, são os que apresentam menor IQA.

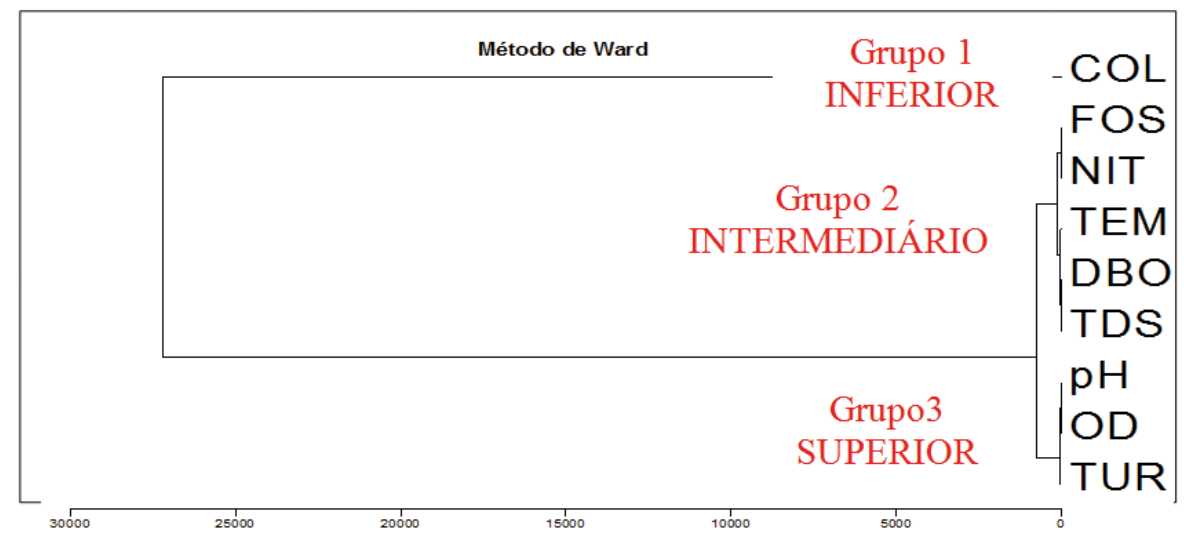

Fig. 2 - Dendograma dos parâmetros amostrados pelo método de Ward. 
O grupo 2 definido como intermediário é representado por dois subgrupos: o primeiro composto pelo Fósforo (FOS) e Nitrato (NIT) e o segundo por Temperatura (TEM), Demanda Bioquímica de Oxigênio (DBO) e Sólidos Totais Dissolvidos (TDS), mesclando o IQA na classificação regular e boa.

178 Entretanto, o grupo 3 caracterizado por superior é representado pelos parâmetros; Potencial Hidrogeniônico (pH), Oxigênio Dissolvido (OD) e Turbidez (TUR), onde todos os pontos desse grupo apresentam maiores valores de IQA em relação aos outros grupos.

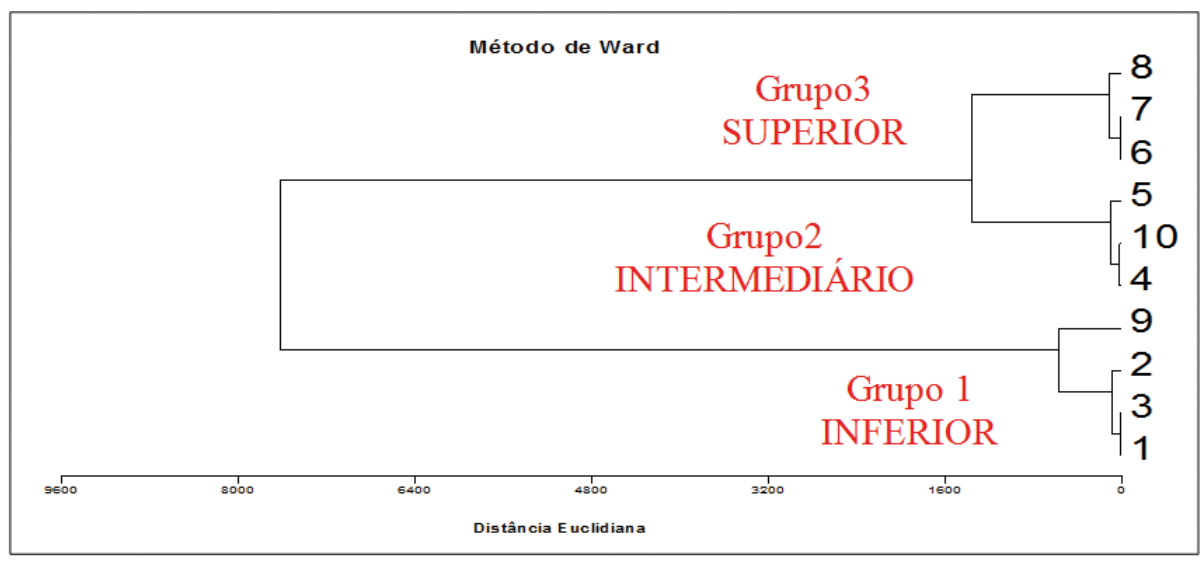

Fig. 3 - Dendograma dos pontos amostrados pelo método de Ward.

O dendrograma dos pontos amostrados pelo método de Ward (Fig. 3) descreve três distintos grupos.

O grupo 1 caracterizado como inferior é composto pelos pontos 9, 2, 3 e 1, sendo todos os pontos enquadrados na classificação de IQA regular. Porém o ponto 9 apresenta características peculiares aos demais pontos, influenciando os demais grupos.

O grupo 2 caracterizado por intermediário, composto pelos pontos 5 e 4 tiveram valores de IQA classificados na faixa considerada regular e o ponto 10 enquadrado na classificação boa.

Já o grupo 3 caracterizado como superior engloba os pontos 6,7 e 8 , sendo todos os valores de IQA também enquadrados na categoria boa, entretanto, apresentam valores mais contundente em relação ao grupo 2 .

O mapa de distribuição dos pontos de IQA pela krigagem dos valores de salinidade, onde os pontos expressos em amarelo são de classificação regular e os pontos expressos em verde possuem classificação boa, tendo sua intensidade caracterizada pelo tamanho das bolas. É possível afirmar que a extensão do rio que sofre maior influencia da salinidade (mais próximo da desembocadura), apresentam boa classificação de IQA, com exceção do ponto 9 que recebe aporte de um pequeno afluente (margeado por uma comunidade) contribuindo diretamente para a redução da qualidade do meio. 

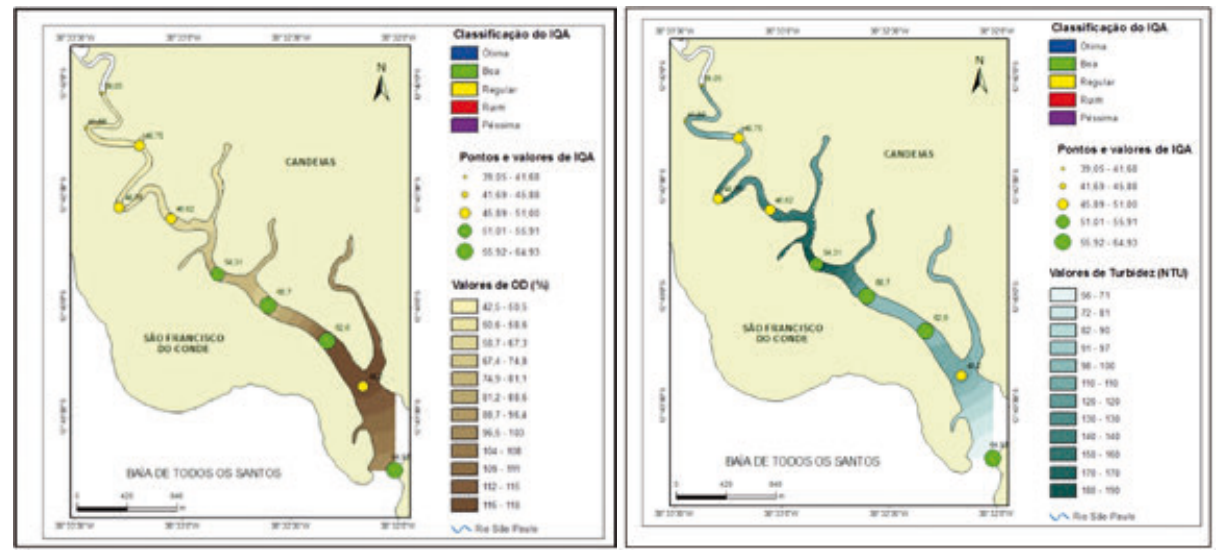

Fig. 4 e 5 - Mapa de distribuição dos pontos de IQA pela Krigagem dos valores de OD(\%) e Turbidez (NTU), respectivamente.

O Oxigênio Dissolvido Saturado (Fig. 4) foi o segundo parâmetro mais representativo, corroborado pela análise no software IQAIDATA, e o mapa de distribuição dos pontos de IQA pela krigagem dos valores de $\mathrm{OD}(\%)$ demonstram que os pontos do rio São Paulo-Ba que apresentam maiores valores de IQA, estão dispostos em uma extensão que sofre maior influencia do $\mathrm{OD}(\%)$ enquadrando na classificação boa. Vale ressaltar que o efluente já anteriormente elucidado, caracteriza o ponto 9, reduzindo a sua qualidade e enquadrando o seu IQA na classificação regular.

O mapa de distribuição dos pontos de IQA pela Krigagem dos valores de Turbidez (Fig. 5), no qual podemos descrever que os pontos de IQA regular são os que possuem maiores valores de turbidez e os pontos que apresentam menor turbidez possuem uma melhor qualidade, com exceção do ponto 9, que mesmo próximo a desembocadura do rio onde a turbidez é menor, se enquadra na classificação de IQA regular.

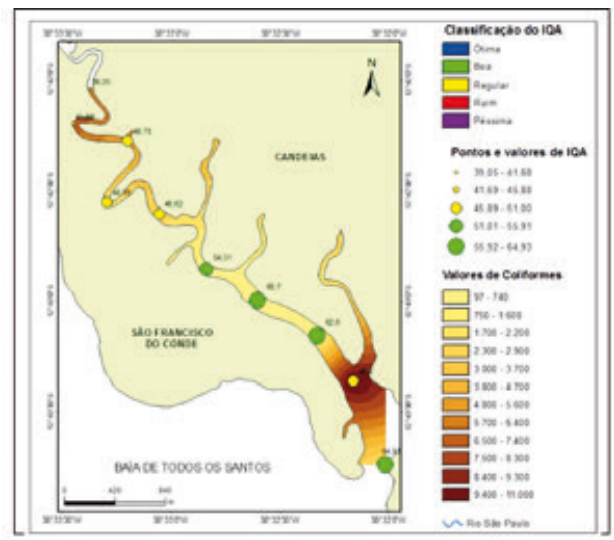

Fig. 6 - Mapa de distribuição dos pontos de IQA pela Krigagem dos valores de Coliformes em NMP/100mL. 
Determinação dos Índices de Qualidade de Água, e a figura 6 caracteriza a distribuiçáo dos pontos de IQA pela krigagem dos valores de coliformes onde a extensão do Rio São Paulo-Ba com maiores concentração dos mesmos são as que apresentam os menores IQAs determinados, dando destaque ao ponto 9 que apresentou o maior valor de Coliformes, reduzindo a sua qualidade.

Desta forma, é possível inferir que o efluente por ser margeado por uma comunidade recebe contribuiçôes domésticas e pode estar propiciando a proliferação e o estabelecimento desse número tâo expressivo dessa variável biológica.

\section{5 - Conclusão}

O Índice de Qualidade de Água (IQA) apresentou uma boa adequação para avaliar a qualidade das águas do rio São Paulo-BA e sua aplicação mostrou que as águas enquadram-se em classes que vão de regular a boa.

Houve uma significativa variabilidade espacial dos valores de IQAs das águas do rio São Paulo.

A análise integrada dos noves parâmetros foi necessária para uma melhor determinação do IQA, possibilitando verificar quais parâmetros foram os mais representativos no índice.

Os coliformes termotolerantes apresentaram valores bem acima da legislação do CONAMA e foi o parâmetro mais representativo na classificação dos Índices de Qualidades das Águas, seguido pelo Oxigênio Dissolvido Saturado e Turbidez, respectivamente.

A análise de Cluster permitiu a formação de diferentes grupos a através de características peculiares as pontos amostrados ao longo do rio São Paulo-Ba, dando ênfase ao ponto 9 que recebe aporte de uma afluente.

As análises geoestatísticas através da krigagem ordinária possibilitam uma melhor visualização da distribuição dos valores de IQA, tornando a interpretação dos dados mais rápida e fácil.

O IQA foi satisfatório para uma avaliação hidroquímica do rio São Paulo-BA, porém expressa de uma forma simplificada uma condição momentânea. Entretanto, O IQA pode ser utilizado para implementação de políticas de gerenciamentos dos Recursos Hídricos se for estabelecido com um sistema de monitoramento.

\section{Referências}

ALMEIDA M. A. B. \& SCHWARZBOLD, A. (2003) - Avaliação sazonal da qualidade daságuas do arroio do Cria Montenegro, RS, com aplicação de um índice de qualidade de água (IQA). Revista Brasileira de Recursos Hidricos 8(1):81-97.

ANDRADE, E. M. et al. (2005) - Índice de qualidade de água: uma proposta para o vale do rio Trussu, Ceará. Revista Ciência Agronômica, v. 36, n. 02, p.135-142.

APHA (2005) - American Public Health Association. Standard methods for the examination of water and watwater, 21st ed. Washington.

BRAGA, B. P. M. \& TUCCI, C. E. M. (1999) - Monitoramento de quantidade e qualidade das águas. Pp. 637-652. In: Rebouças, A. C., Braga, B. \& Tundisi, J. G. (Eds). Águas Doces no Brasil. Escrituras, São Paulo, 717p. 
CIMITESINOS (1990) - Comitê de presevarção, gerenciamento e pesquisa da bacia do rio dos sinos. Utilizando de um índice de qualidade da água no rio dos Sinos. PortoAlegre. p. 33.

CETESB (2003) - Companhia de tecnologia de saneamento ambiental. Relatório de qualidade das águas interiores do Estado de São Paulo, 2002. São Paulo. p. 274.

CETESB (2004) - Companhia de tecnologia de saneamento ambiental. Relatório de qualidade das águas interiores do Estado de São Paulo, 2003. São Paulo. p. 273.

KONIG, R., SUZIN, C. R. H., RESTELLO, R. M. \& HEPP, L. U. (2008) - Qualidade das águas de riachos da regiâo norte do Rio Grande do Sul (Brasil) através de variáveis físicas, químicas e biológicas. Pan-American Journal of Aquatic Sciencs.

LOPES, F. B., TEIXEIRA, A. S., ANDRADE, E. M., AQUINO, D. N. \& ARAÚJO, L. F. P. (2008) - Mapa de qualidade das águas do rio Acaraú, pelo emprego do IQA e Geoprocessamento. Centro de Ciências Agrárias, UFC, Fortaleza-CE. Revista Ciência Agronômica, v.39, n.3, p. 392-402.

MELO JUNIOR, G., COSTA, C. E. F. S. \& CABRAL NETO, I. (2003) - Avaliaçáo hidroquímica e da qualidade das águas de um trecho do rio Açu, Rio Grande do Norte. Revista de Geologia, v. 16, n. 02, p. 27-36.

PEKEY, H., KARAKAS, D. \& BAKOGLU, M. (2004) - Source apportionment of metals trace in surface waters of a polluted stream using multivariate statistical analyses. Marine Pollution Bulletin, 49.p. 809-818.

PIASENTIN, A. M., SEMENSATTO JUNIOR, D. L., SAAD, A. R., MONTEIRO JUNIOR, A. J. \& RACZKA, M. F. (2009) - Índice de qualidade da água (IQA) do reservatório Tanque Grande, Guarulhos (SP): Análise sazonal e efeitos do uso e ocupação do solo. São Paulo, UNESP, Geociências, v.28, n.3, p. 305-317.

POMPEU, P. S., ALVES, C. B. M. \& CALLISTO, M. (2005) - The effects of urbanization on biodiversity and water quality in the Rio das Velhas Basin, Brazil. American Fisheries Society Symposium, 42: 11-22.

Simóes F. S., Yabe M. J. S., Moreira A. B. \& Bisinoti M. C. (2007) - Avaliação do efeito da piscicultura em sistemas aquáticos em Assis e Cândido Mota, São Paulo, por indicador de qualidade da água e análise estatítica multivariada. Quim. Nova, 30(8): 1835-1841.

STOLFI, C. M. \& FIGUEREIDO, B. R. (2008) - Parâmetros de referência para estudos de qualidade de águas em área de remanescente de Mata Atlântica urbano. Departamento de Geologia e Recursos Naturais, Instituto de Geociências, UNICAMP. Campinas- SP.

THORNE, R. S. J. \& WILLIAMS, W. P. (1997) - The response of benthic macro invertebrates to pollution in developing countries: a multimetric system of bioassessment. Freshwater Biology, 37: 671-686. 